\title{
Modelos estadísticos de ruido ambiental para el Distrito Metropolitano de Quito DMQ, mediante datos históricos del 2009 al 2015, validados al 2019, como herramienta de calidad ambiental
}

\author{
Katty Coral ${ }^{1,2^{*}}$, Tania Moromenacho ${ }^{1}$, Alonso Moreta ${ }^{1}$, Fabio Villalba', Jorge Esteban Oviedo ${ }^{2}$ \\ 'Universidad Internacional SEK (UISEK), Facultad de Ciencias Naturales y Ambientales, Alberto \\ Einstein y 5 ta transversal, Campus Miguel de Cervantes, Quito Ecuador \\ ${ }^{2}$ Universidad de Cantabria, Escuela Técnica Superior de Ingenieros Industriales y \\ Telecomunicaciones, Grupo de Investigaciones QuiPre. Av. De los Castros S/N Santander España \\ *Autor principal / Corresponding Author, e-mail: katty.coral@uisek.edu.ec
}

\section{Statistical models of environmental noise for the Metropolitan District of Quito, using historical data from 2009 to 2015, validated to 2019, as an environmental quality tool}

\begin{abstract}
The management of the traffic noise within the Metropolitan District of Quito has been tried indirectly, only as an indicator of air pollution control. However, studies have forgotten the evaluation of human health effects and the magnitude of the polluting agent in the city. The increase of vehicles of $12 \%$ annually has saturated the roads, and the traffic noise is more in an urban zone at the District. For this reason, the present project had the objective of designing statistical models of traffic noise in DMQ with historical data from 2009-2015, and its subsequent verification with data from the Environment Secretary of the DMQ to 2019, applying simple and multiple linear regression analysis. A database and an analysis of the behavior of noise in recent years carried out, influenced by the traffic flow in the city, establishing a relationship between the number of vehicles and the equivalent noise. Finally, the validation and evaluation of the models performed employing confidence intervals and least squares regression by calculating the correlation coefficient of these models and validating them through the analysis of variances (ANOVA) that allowed determining the magnitude of the relationship between the variables and the effectiveness of the model. The result from the investigation determined the noise maintains a variant tendency, which is related to the number of vehicles circulating in the streets of Quito as well as the speed of the same ones. It's evident that during the day the noise frequently changes until six o'clock in the evening and from seven o'clock at night, the noise decreases as a function of the diminution of the vehicles. For that reason, the simple linear regression models, as well as the zonal and integral multiple models, adjust to the conditions of the urban area South, Center, and North of the Metropolitan District of Quito.
\end{abstract}

Keywords: Confidence interval, Correlation coefficient, Linear regression model, Multivariate analysis, Traffic noise 


\section{Resumen}

La gestión del ruido generado por el flujo vehicular en el Distrito Metropolitano de Quito (DMQ) se ha tratado de forma indirecta, es decir únicamente como medida de control de la calidad de aire, lo cual no está mal. Sin embargo, se ha dejado de lado el estudio para evaluar los efectos generados en la población y la magnitud del contaminante en la ciudad ocasionado por el crecimiento desmedido del parque automotor del 12\% anual, hecho que satura las vías e incrementan la contaminación acústica. Por tal razón, el presente proyecto tuvo por objetivo diseñar modelos estadísticos de ruido de tráfico rodado en el DMQ con datos históricos del 2009-2015, aplicando el análisis de regresión lineal simple y múltiple, y su posterior comprobación con datos de la Secretaría de Ambiente del DMQ al 2019. Para lo cual se procedió a establecer una base de datos y un análisis del comportamiento del ruido en los últimos años influenciado por el flujo vehicular en la ciudad, estableciendo la relación entre la cantidad de vehículos y el ruido equivalente. Finalmente se realizó la validación y evaluación de los modelos mediante intervalos de confianza y la regresión por mínimos cuadrados calculando el coeficiente de correlación de dichos modelos y validándolos mediante el análisis de varianzas (ANOVA), que permitieron determinar la magnitud de relación entre las variables y la efectividad del modelo. El resultado de la investigación determinó que el ruido mantiene una tendencia variante, la cual está relacionada con el número de vehículos que circulan por las calles quiteñas así como la velocidad de los mismos, es evidente que durante el día el ruido cambia constantemente hasta las seis de la tarde y a partir de las siete de la noche el ruido decrece en función a la disminución de los vehículos. Por tal razón, los modelos de regresión lineal simple así como el múltiple, zonales e integrales, se ajustan a las condiciones de la zona urbana Sur, Centro y Norte del Distrito Metropolitano de Quito.

Palabras clave: Análisis multivariante, Coeficiente de correlación (R2), Intervalo de confianza, Modelo de regresión lineal simple, Ruido de tráfico vehicular,

\section{INTRODUCCIÓN}

El ruido es un sonido desagradable para quien lo percibe [1] y que de acuerdo al tiempo de exposición puede causar efectos fisiológicos, psicológicos y sociales no deseados, convirtiéndose en un contaminante que tiende a perturbar las distintas actividades humanas como el sueño, el descanso, el trabajo, el estudio, entre otros; creando un estado de cansancio y tensión, degradando la calidad de vida y salud de las personas [2].

De acuerdo a las estadísticas de la Organización Mundial de la Salud [1], la sordera es un efecto común de la exposición al ruido, ya que este contaminante únicamente se percibe con el sentido del oído. Aun cuando depende de la percepción de cada individuo, al llegar a un volumen determinado las personas presentan molestias e irritación.

El impacto ambiental generado por el ruido vehicular generalmente pasa desapercibido por las autoridades ambientales, principalmente por la propiedad emergente de sus efectos [3]. De acuerdo a las investigaciones realizadas en varios países, se ha demostrado que la principal fuente de contaminación acústica proviene del transporte vehicular, producto de la movilización diaria de las personas desde sus hogares hacia su sitio de 
trabajo o estudio, sobrepasando la capacidad de las vías y llegando a alcanzar niveles de ruido entre 80 y $90 \mathrm{~dB}$ (A), sonido comparable con la de un taladro neumático [4].

Los primeros modelos enfocados a medir y predecir el ruido generado por los vehículos fueron diseñados en países desarrollados de la Unión Europea. Varios de ellos, han servido de guía para países de América Latina como el Modelo RLS-90 (Alemania), Modelo CERTU (Francia), Modelo STL-86 (Suiza), Modelo CORTN (Gran Bretaña), Modelo SÁNCHEZ (España) [5]. En el caso específico del Distrito Metropolitano de Quito, los dos últimos modelos fueron validados en los años 2012, 2013, mediante investigaciones realizadas por la Universidad Internacional SEK, $[6,7]$ sin obtenerse datos que garantizaran su aplicación a la ciudad.

Este estudio se enfocó en determinar y validar las ecuaciones estadísticas de ruido para las zonas sur, centro y norte del Distrito Metropolitano de Quito, así como la determinación de un modelo integral para la ciudad, capaz de evaluar el ruido con respecto a la influencia de las variables independientes velocidad, flujo vehicular liviano y pesado. Se desecharon elementos constructivos como calles, fachadas, tipo de vía carrozable, altura de edificios, con el fin de hacer al modelo amigable y de fácil aplicación al disponer de variables relativamente sencillas de cuantificar en el día a día.

De acuerdo a la Secretaría del Ambiente del Distrito Metropolitano de Quito, los niveles de ruido máximos que se han medidos son de $78 \mathrm{~dB}(\mathrm{~A})$ en el día, valor que supera los límites sugeridos por la Organización Mundial de la Salud OMS para ambientes exteriores de $65 \mathrm{~dB}(\mathrm{~A})$ en el mismo lapso de tiempo. Esto significa que la calidad de vida en la ciudad no es óptima, además se conoce que el crecimiento del parque automotor es del $12 \%$ anual [8,34], los cuales saturan la capacidad de las vías y consecuentemente el tráfico vehicular genera emisiones de ruido intensas. Pero suponer no define un diagnóstico certero del comportamiento del ruido, ni tampoco se puede proyectar a futuro, por tal razón aplicar un modelo estadístico con un coeficiente de correlación adecuado permite realizar análisis que pueden simular la realidad local y definir el comportamiento de la variable dependiente $\left(L_{e q}\right)$ con relación a las variables independientes (velocidad y flujo vehicular), simplificando su monitoreo, uno de los objetivos claves del presente estudio.

El estudio del ruido es complejo en relación a otros contaminantes, porque no presenta efecto acumulativo en el medio, no se traslada a través de los sistemas naturales, e incluso no deja residuos. Sin embargo mediante los muestreos consecutivos y los datos recopilados se ha comprobado que usando conceptos estadísticos se puede llegar a determinar modelos predictivos de ruido, que permitan simular la realidad del lugar mediante una ecuación estadística, con mediciones sencillas de volumen de tráfico, generando un ahorro de recursos económicos considerables [9].

En el Distrito Metropolitano de Quito (DMQ) circulan aproximadamente 540827 vehículos [35], de los cuales el 77\% corresponden al transporte liviano particular. El crecimiento vehicular en el DMQ es una manifestación de la coexistencia entre las diferentes zonas y las realidades físico-espaciales del lugar, debido al movimiento poblacional desde los Valles (Los Chillos y Cumbayá) hacia el hipocentro de la ciudad de Quito, lugar en donde se encuentran las áreas educativas, residenciales, industriales y lo más importante el desarrollo del comercio [10]. 
El objetivo del proyecto radicó en diseñar los modelos estadísticos de ruido ambiental generados por el flujo vehicular para la ciudad de Quito, mediante datos históricos, como herramienta de calidad ambiental; para ello fue necesario generar una base de datos con información preexistente (trabajos de fin de carrera de Ingeniería Ambiental de la Universidad Internacional SEK) desde el año 2009 al 2015 [6,7,14-32], para ser utilizada en la elaboración de los modelos, obtener ecuaciones estadísticas de ruido ambiental generado por tráfico vehicular para cada zona muestreada: sur, centro y norte de la Ciudad de Quito y dos ecuaciones integradas (generales) para toda la ciudad, utilizando el método de linealización de una función no lineal y análisis multivariante; esto con el fin de que sean aplicadas como herramienta de calidad ambiental, para ello fue necesario determinar el nivel de ajuste y aceptación de los modelos estadísticos obtenidos. Los datos son un reflejo del flujo vehicular exclusivamente, minimizando los efectos de fuentes fijas, pues entre el ruido ambiental es producto mayoritariamente del ruido vehicular y es una combinación del ruido de fondo y del tráfico rodado, de ahí que los modelos estadísticos corresponden a la medición de estos dos factores. [36]. Adicionalmente tanto el Texto Unificado de Legislación Ambiental Secundaria del Ecuador TULAS [38], como el Código Único Municipal del DMQ [37], regulan y controlan las fuentes fijas de emisión de ruido, por lo que su aporte al ruido ambiental es aún menor.

\section{MATERIALES Y MÉTODOS}

La investigación se realizó en base a la recopilación de 21 estudios anteriores, consistentes en monitoreos de ruido ambiental, en periodos y zonas distribuidas a lo largo y ancho del área urbana de la ciudad de Quito y mapeo de los valores obtenidos, lo que ha permitido determinar la tendencia de comportamiento del ruido y generar los modelos desde el punto de vista estadístico. Los resultados obtenidos reflejan directamente el ruido generado por el tráfico vehicular y el ruido de fondo.

\section{Mediciones de Ruido}

Los datos obtenidos de ruido ambiental en los estudios tomados de referencia y realizados en la UISEK desde el año 2009 hasta el 2019, se utilizaron sonómetros integradores tipo 1 y calibradores de ruido previamente calibrados, y siguiendo lo establecido por la Normativa Ecuatoriana, en el TULAS hasta el 2015, donde entró en vigencia el Acuerdo Ministerial 097 A.

\section{Manejo de Datos}

Se recopilaron datos experimentales sin promediar de nivel de presión sonora (NPS), velocidad promedio y flujo vehicular: Livianos, Pesados y Total desde el año 2009 al 2015, los cuales se caracterizan por ser 126 datos numéricos por cada variable, correspondientes a 18 horas diarias muestreadas (6h00 a 00h00) para cada día de la semana (de Lunes a Domingo). Los valores numéricos de las variables (Velocidad, NPS y flujo vehicular) se organizaron en hojas de cálculo para cada zona (Sur, Centro y Norte), diferenciándolos para los siete días de la semana (de lunes a domingo). La organización de los datos se realizó en un formato en el que la velocidad promedio se representa por $V_{\text {prom }}$ y se expresa en $\mathrm{km} \cdot \mathrm{h}^{-1}$; el flujo vehicular $\left(Q . h^{-1}\right)$ es el número de vehículos contabilizados en 
una hora, la cual se disgrega en livianos $\left(Q_{Q}\right)$, pesados $\left(Q_{p}\right)$ y los vehículos totales $\left(Q_{t}\right)$ y finalmente el nivel sonoro NPS $\left(L_{e q}\right)$ expresado en dB $(A)$.

Se limitaron las zonas de estudio de acuerdo a la distribución de los puntos monitoreados a lo largo de las calles principales del área urbana del DMQ, definiendo los barrios urbanos que cruzan las calles conforme al mapa Parroquias Rurales del Distrito Metropolitano de Quito [33]. En la Figura 1, se aprecia los puntos monitoreados en la ciudad.

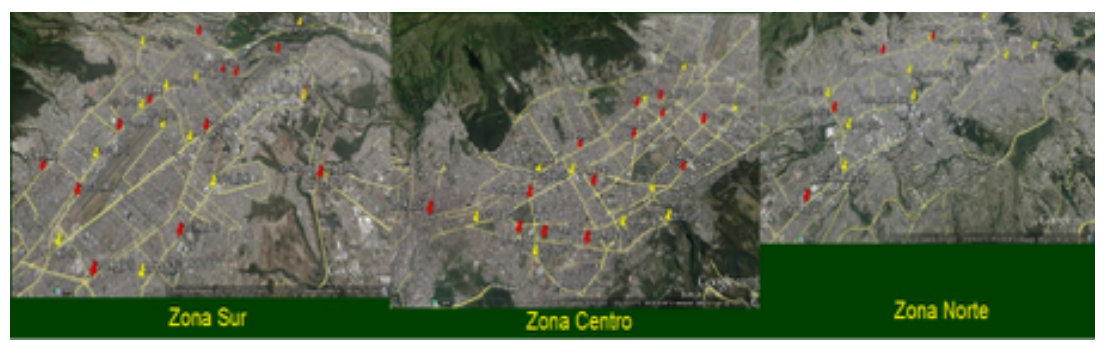

FIGURA 1. Puntos de muestreo en el DMQ para la obtención de datos primarios

\section{Análisis de frecuencia de los datos numéricos}

Se elaboraron tablas de frecuencia para cada zona con el fin de determinar el número de observaciones que más se repiten para interpretar el comportamiento del nivel de Presión Sonora $\left(L_{e q}\right)$ en el periodo 2009-2015. Se establecieron número de observaciones, valores máximos y mínimos, el rango calculado mediante la diferencia entre el valor máximo y mínimo; el número de clases se determinó con la desigualdad $2^{c}>n$, donde c es el número de clases y $n$ es el número de observaciones; y por último el ancho del intervalo de clase que se calcula dividiendo el rango para el número de clases calculado.

\section{Diseño del modelo estadístico predictivo de ruido urbano para la ciudad de Quito}

El proyecto se fundamentó en el análisis estadístico de las variables provenientes de la base de datos 2009-2105, para lo cual se utilizaron las siguientes variables:

Vehículos pesados $\left(Q_{p}\right)$

Vehículos ligeros $(Q)$

Velocidad promedio $\left(V_{\text {prom }}\right)$

Nivel de presión sonora equivalente $\left(L_{e q}\right)$

\section{Determinación del Coeficiente de Correlación Lineal Simple (r)}

Mediante este estadístico se analizó el grado de asociación lineal entre la variable del nivel de ruido y el flujo vehicular. Los valores que se evaluaron oscilan entre 1 y -1, lo cual demuestra la asociación ya sea positiva o negativa según sea el caso, la principal observación que se consideró es que el valor determinado no sea cercano a 0, ya que significaría que no existe asociación lineal y por ende se descartaría la posibilidad de realizar el modelo lineal. 


\section{Modelo Estadístico predictivo de ruido urbano a partir de regresión lineal simple. RL}

Con las tablas consolidadas se procedió a diseñar el modelo estadístico, para el cual se determinó las constantes a y b de la ecuación de regresión lineal simple. [34]

Donde:

$$
Y=a+b X_{1}
$$

$Y=$ variable dependiente

$X_{1}=$ variable independiente

De acuerdo a la experiencia de trabajos anteriores, se considera que la ecuación de regresión lineal simple para ruido es la siguiente:

$$
L_{e q}=a+b \log Q_{t}
$$

Donde:

$L_{e q}=$ es el nivel de ruido equivalente en $\mathrm{dB}(\mathrm{A})$.

$a$ y $b=$ constantes propias de la región obtenidos en base a los datos experimentales monitoreados.

$Q_{t}=$ es el flujo vehicular total (suma vehículos livianos y vehículos pesados)

Con estos antecedentes, se procedió a realizar la regresión lineal simple para cada Zona (Sur, Norte y Centro), identificando la ecuación ajustada y el porcentaje de correlación para cada caso.

\section{Modelo Estadístico predictivo de ruido urbano a partir de Análisis Multivariante. MV}

El desarrollo del análisis multivariante consistió en realizar una regresión de tres variables independientes (velocidad, flujo vehicular liviano y flujo vehicular pesado) y una variable dependiente (nivel de ruido). Para lo cual se consolidaron los datos en una tabla resumen, esto permitió tener datos representativos con respecto al grupo de valores de ruido ambiental en esos años, e incluso se reduce el grado de dispersión de los datos al momento de desarrollar la regresión.

Los datos utilizados fueron:

$L_{e q}(A)$ : Nivel de ruido equivalente en $\mathrm{dB}(\mathrm{A})$, consolidados a ocho datos por hora.

Q: número de vehículos livianos por hora.

$Q_{p}$ : número de vehículos pesados (buses, camiones, etc.)

$\log Q_{1}$ y $\log Q_{p}$ : logaritmo base 10 del flujo vehicular

$V_{\text {prom }}$ : velocidad promedio $\left(\mathrm{km} \cdot \mathrm{h}^{-1}\right)$

El modelo de regresión lineal múltiple es idéntico al modelo de regresión lineal simple, con la única diferencia de que aparecen más variables explicativas [11]. La ecuación generalizada del análisis multivariante es la siguiente:

$$
Y=a+b_{1}\left(X_{1}\right)+b_{2}\left(X_{2}\right)+b_{3}\left(X_{3}\right)+b_{4}\left(X_{4}\right)
$$


Mientras que la expresión utiliza en determinación de ruido es:

$$
L_{\text {eqhora }}=a+b \log Q_{1}+c \log Q_{p}+d V_{\text {prom }}
$$

En donde:

$a, b, c$ y d: son los coeficientes constantes correspondientes a cada variable. $Q_{\mu} Q_{p^{\prime}}$ y $V_{\text {prom }}$ : son las variables independientes.

\section{Validación de los modelos determinados}

La validación de los modelos de regresión lineal simple y análisis multivariante se realizó mediante la estimación del intervalo de confianza y el análisis ANOVA. Los valores utilizados para validación fueron los valores promedios para cada zona obtenidos a lo largo del periodo de investigación.

\section{Análisis de Intervalo de confianza}

La validación por el análisis del intervalo de confianza, se realizó mediante una tabla comparativa entre el valor de ruido equivalente experimental y el calculado.

- Media: es la media aritmética de los niveles de presión sonora calculados y experimentales.

- Desviación Estándar: permite determinar cuan alejados están los datos con respecto a la media.

- Coeficiente de variación: se determinó con la división entre la desviación estándar y la media.

- Coeficiente de correlación: se realizó aplicando la fórmula del coeficiente de correlación de dos grupos de datos, se comparó la relación existente entre el nivel de ruido experimental y las variables independientes de velocidad, flujo vehicular liviano, pesado y total.

Finalmente se determinó el nivel de confianza del 99\% para calcular el intervalo, de tal forma que se procedió a buscar el valor correspondiente a este porcentaje en la tabla T-de Student y aplicar la siguiente ecuación:

$$
L C=X m \pm t_{n-1} \frac{S}{\sqrt{n}}
$$

Donde:

$X$ media $=$ dependiente de cada Estación

$n$ número de datos $=$ para todos los casos 18 (horas)

S desviación estándar = dependiente de cada Estación

Nivel de confianza $=99 \%=>t_{n-1}=2,8982$

\section{Análisis ANOVA}

Para establecer si existen diferencias significativas entre los valores de ruido ambiental experimentales y los calculados a través de las ecuaciones estadísticas establecidas, se utilizó el análisis ANOVA, estableciendo como hipótesis nula que los niveles de ruido 
calculados vs los experimentales, no tienen una diferencia significativa, pudiendo esta diferencia ser asignada a un error de medición ya sea humano o de los equipos utilizados, permitiendo aceptar las ecuaciones zonales y generales como apropiadas para la predicción de ruido ambiental a través de la determinación del número de automóviles circulantes por un determinado sector y la velocidad de los mismos.

Para el análisis de las varianzas se plantearon las dos hipótesis sugeridas por la metodología, la hipótesis nula hace énfasis en que todas las medias son iguales y la hipótesis alternativa que plantea que por lo menos una de las medias es diferente. El rango de entrada en este caso son: el valor experimental y los valores calculados. El valor alfa se mantiene en 0,05.

El análisis ANOVA se lo realizó para cada zona establecida del DMQ: Sur, Norte y Centro; comparando las medias de los valores experimentales (obtenidos en campo) con los valores zonales de RL, MV y los valores obtenidos de las ecuaciones integrales RL y MV de la ciudad, con el fin de determinar la validez de cualquiera de las ecuaciones para la predicción del ruido ambiental en la ciudad de Quito.

\section{Comprobación 2019}

Debido al tiempo transcurrido entre la elaboración de las ecuaciones de ruido ambiental producido por tráfico vehicular, se decidió tomar muestras de las variables involucradas en los modelos durante el año 2019, escogiendo para la comparación, una semana completa de lunes a domingo, de $6 \mathrm{~h} 00$ a 00h00, una vez al mes. Para esto se escogió los puntos de monitoreo de ruido ambiental establecidos por la Secretaría de Ambiente del DMQ desde el año 2015, valores validados por el personal técnico de la Secretaría y por lo tanto confiables a cabalidad. A pesar de que la Secretaria dispone de cuatro puntos de muestreo permanentes, las 24 horas del día, en Jipijapa, El Camal, Carapungo y Centro, se escogió para la comparación los datos correspondientes a las horas y semanas correspondientes al muestreo paralelo realizadas por la UISEK en tres sitios representativos a las zonas donde se desarrollaron las ecuaciones respectivas. En el Centro en las coordenadas $78^{\circ} 30^{\prime} 36^{\prime \prime}$ W, $0^{\circ} 13^{\prime} 12^{\prime \prime} \mathrm{S}$, a una altura referencial de 2820 msnm, en la García Moreno 751 y Sucre, zona que para el año 2019 se encontraba peatonalizada, circulando exclusivamente los automóviles correspondientes a las entidades gubernamentales de la zona. En el Sur en el punto El Camal, ubicado en las coordenadas 78 $30^{\prime} 36^{\prime \prime}$ W, $0^{\circ} 15^{\prime} 00^{\prime \prime}$ S, a 2840 msnm al ingreso del Patronato Municipal San José Sur , en las calles Adrián Navarro 1660 e Hinostroza. Por último, en el norte se escogió la ubicación Carapungo, ubicado a 78²6'50"W, 05'54"S, altura de 2660 msnm, en la Super manzana B, el Verjel S/N, edificio Andinatel. Ninguno de los sitios escogidos presenta alto tráfico vehicular, de hecho podrían considerarse de medio y bajo fluido automotriz. Para la comparación, la Secretaría de Ambiente del DMQ proporcionó los datos horarios (24 horas) tabulados para todos los días del año, las 24 horas del día. Con estos datos se realizó el promedio de ruido para cada día de la semana escogida mensualmente, las 12 horas contabilizadas y con la ecuación (6) se procedió a calcular los promedios semanales de Leq.

$$
\text { Leq }=10 \log _{10}\left(\frac{1}{T}\right)\left(\sum_{i=1}^{N} \Delta P_{i} 10^{\left(\frac{\text { Leqi }}{10}\right)}\right)
$$


Dónde:

Leq = Nivel de presión sonora continuo equivalente

Leqi $=$ Nivel de presión sonora equivalente instantánea

$\Delta P i=$ fracción de tiempo de la medición [39].

\section{RESULTADOS Y DISCUSIÓN}

\section{Modelos predictivos de ruido para la Zona Sur}

Con los datos procesados en la Zona Sur, correspondiente al periodo 2009-2015, se establecieron como calles principales la Av. Mariscal Sucre, Av. Morán Valverde y Av. Napo, considerando como punto delimitante la loma del Panecillo. Este tramo abarca los barrios urbanos La Magdalena, Chimbacalle, San Bartolo, La Ferroviaria, Solanda, La Argelia y Quitumbe. El total de datos tabulados para la Zona Sur fueron de 2016 por cada variable, que incluyen una variable dependiente (NPS) y cuatro variables independientes $\left(V_{\text {prom }} Q_{\mu}, Q_{p}\right.$ y $\left.Q_{t}\right)$.

A partir del estudio de frecuencias, se determinó que no existen una cantidad de datos relevantes menores a 70,0 dB (A), sin embargo es frecuente encontrar valores mayores a $71,9 \mathrm{~dB}(\mathrm{~A})$, siendo los más frecuentes $71,9 \mathrm{~dB}(\mathrm{~A})$ y $74,9 \mathrm{~dB}(\mathrm{~A})$ representados por el $66 \%$ de los datos. Los valores que llegaron a superar los 75,0 dB (A) representan el 19\%, esto significa que existe la posibilidad de incremento en la emisión de ruido en el sector con el tiempo y con el crecimiento del parque automotor.

La ecuación obtenida y validada para el modelo de regresión lineal simple de la Zona Sur para el día y noche, hasta las 00H00, del DMQ se reporta en la ecuación 7, con un coeficiente de correlación de 0,96:

$$
L_{\text {eqhora }}=2,63+20,12 \log Q_{t}
$$

El análisis multivariante se realizó con los datos de flujo vehicular liviano y pesado, desde las seis de la mañana hasta las $00 \mathrm{H} 00$, expresados en logaritmo base diez y la velocidad promedio en $\left(\mathrm{kmh}^{-1}\right)$. La ecuación obtenida y validada para el modelo de análisis multivariante para la Zona Sur del DMQ, se presenta en la ecuación 8, con un coeficiente de correlación múltiple de 0,99:

$$
L_{\text {eqhora }}=24,12+7,13 \log Q_{1}+6,77 \log Q_{p}+0,18 V_{\text {prom }}
$$

En la Figura 2, se observa que los datos calculados con la ecuación 6 presentan una mejor predicción de los valores en el periodo de la tarde (12H00 a 18H00), además el error de predicción de la misma ecuación, indica que para este periodo es cero, es decir que el modelo de predicción lineal simple se ajusta mejor con valores promedio de flujo vehicular de 2784 automóviles, con una desviación estándar de 68 vehículos. 


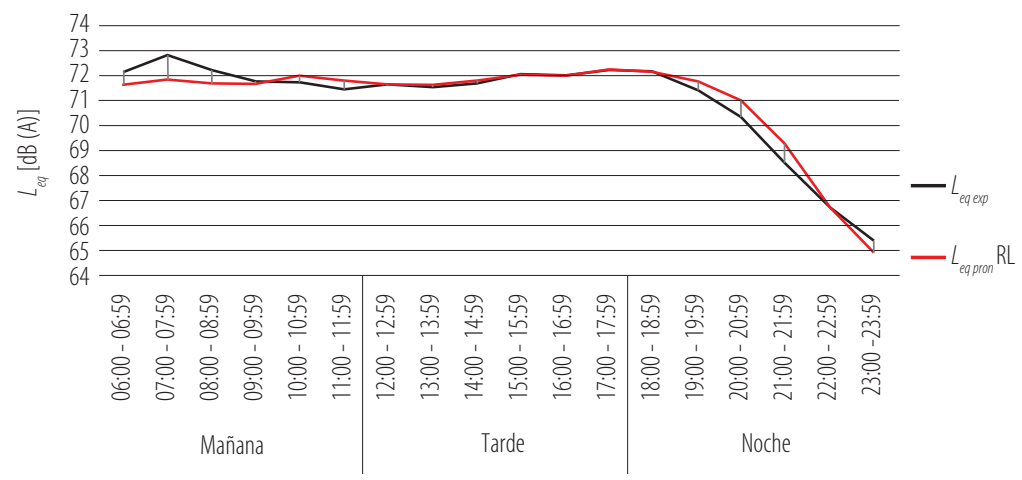

$L_{\text {eq pron }}$ : nivel de presión sonora equivalente pronosticado

$L_{\text {eqexp }}$ : nivel de presión sonora equivalente experimental

RL: regresión linea

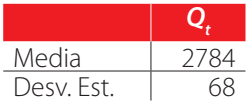

FIGURA 2. Comparación $L_{\text {eqexp }}$ vs. $L_{e q}$ RL para la Zona Sur

Los datos calculados con la ecuación 7, se ajustan de forma global a las condiciones de la Zona Sur. Sin embargo, al integrar tres variables, el método de ajuste demanda mayor trabajo y condicionamientos estrictos para cada variable. De tal forma que la velocidad debe estar dentro del rango 37-38 $\mathrm{kmh}^{-1}$, el flujo vehicular liviano (Q) entre 1913 - 2677 vehículos livianos por hora y el transporte pesado $\left(Q_{p}\right)$ en el rango de 180 y 350 vehículos pesados por hora.

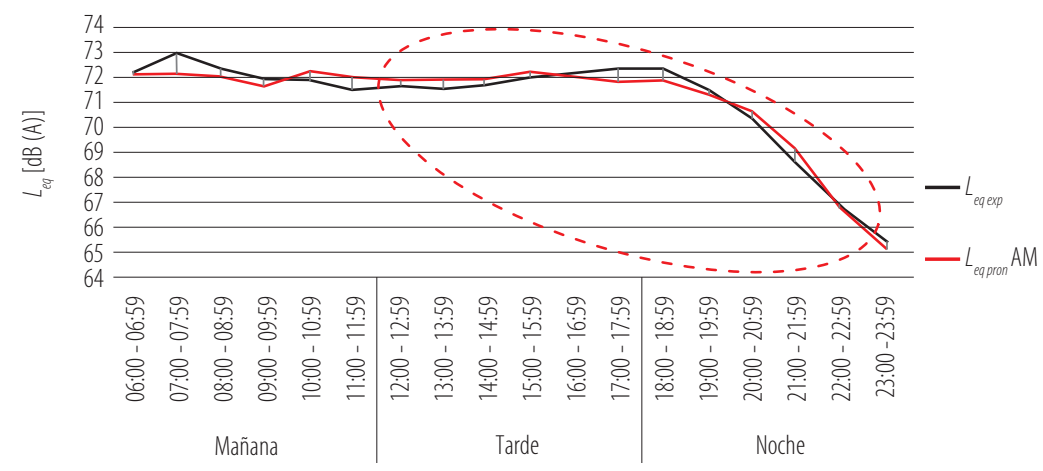

\footnotetext{
$L_{\text {eq pron }}$ : nivel de presión sonora equivalente pronosticado $L_{\text {eqexp }}$ : nivel de presión sonora equivalente experimental RL: regresión linea
}

\begin{tabular}{l|r|r|r} 
& $\boldsymbol{V}_{\text {prom }}$ & \multicolumn{1}{|c|}{$\mathbf{Q}_{\mathbf{1}}$} & $\mathbf{Q}_{\mathbf{p}}$ \\
\hline Media & 37 & 2295 & 265 \\
\hline Desv. Est. & 1 & 382 & 85
\end{tabular}

FIGURA 3. Comparación $L_{\text {eqexp }}$ vs. $L_{\text {eq }}$ AM para la Zona Sur 


\section{Dodelos predictivos de ruido para la Zona Centro}

El punto limitante es la Loma del Panecillo y las calles principales de monitoreo son Av. 12 de Octubre, Av. 10 de Agosto, Av. América y Av. 6 de Diciembre, las cuales cruzan los barrios urbanos Rumipamba, Iñaquito, Jipijapa, Belisario Quevedo y Mariscal Sucre. El total de datos tabulados para la Zona Centro fue de 2772.

De acuerdo a los datos experimentales analizados, el ruido en la Zona Centro no presenta valores menores a 70,0 dB (A) y es muy frecuente que existan valores mayores a $72,6 \mathrm{~dB}(\mathrm{~A})$ pudiendo llegar a un máximo de $75.5 \mathrm{~dB}(\mathrm{~A})$. Sin embargo los niveles de ruido recurrentes fluctúan entre $72,6 \mathrm{~dB}(\mathrm{~A})$ y $75,5 \mathrm{~dB}(\mathrm{~A})$ representando el $68 \%$ de los datos y un $15 \%$ de valores mayores a $78,5 \mathrm{~dB}(A)$; este dato se justifica por el incremento de vehículos en horas pico.

Para la obtención del modelo estadístico por linealización del ruido ambiental de la Zona Centro, se realizó el análisis de los datos consolidados en 18 valores representativos para todo el día. La Ecuación 8 establece la relación obtenida y validada con los datos monitoreados:

$$
L_{\text {eqhora }}=35,7+11,16 \log Q_{t}
$$

El coeficiente de correlación del nivel de ruido equivalente con el flujo vehicular total es de 0.98 , lo que significa una representación relevante.

La ecuación para el análisis multivariante de la Zona Centro, obtenida bajo iguales premisas que en la Zona sur, se refleja en la Ecuación 10:

$$
L_{\text {eqhora }}=21,33+11,58 \log Q_{1}+3,32 \log Q_{p}+0,18 V_{\text {prom }}
$$

En el caso de la Zona Centro, el número de vehículos pesados por hora $\left(Q_{p}\right)$ tiene un coeficiente de 0,92, seguido por 0,98 del flujo vehicular liviano $(Q)$, pero algo importante sucede con la velocidad promedio $\left(V_{\text {prom }}\right)$ este es un valor negativo de - 0,93, esto significa que la relación es inversamente proporcional. Por tanto las tres variables tienen una intensidad de relación significativa, siendo la correlación combinada igual a 0,98.

En la Figura 4 se aprecia la relación entre los valores experimentales y los obtenidos a través de la Ecuación 8, presentando una mejor predicción de los valores en el periodo de la tarde y noche, además el error de predicción de la misma ecuación, indica que para estos periodos es 0,2 y 0,1 respectivamente. Es decir que el modelo de predicción lineal simple, para lo Zona Centro del DMQ, se ajusta mejor con valores promedio de flujo vehicular de 2024 vehículos. 


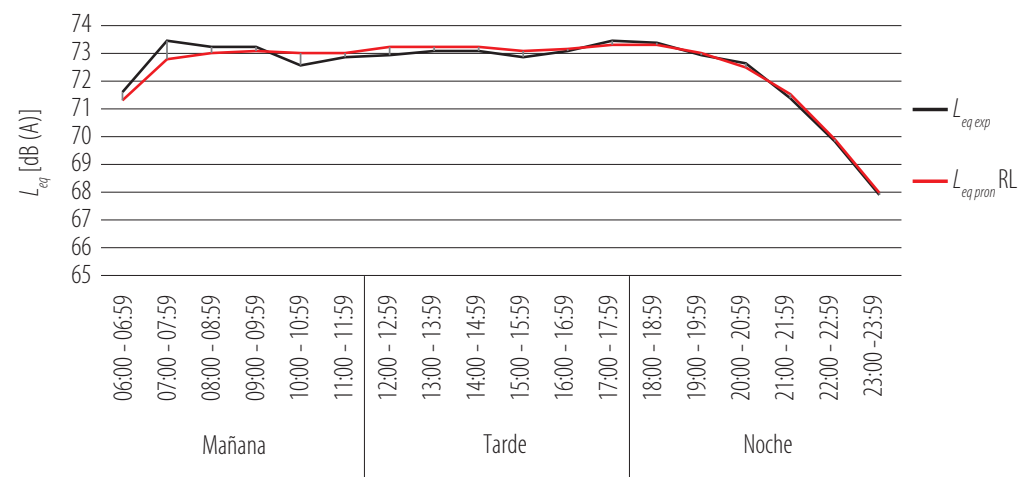

$L_{\text {eq pron }}$ : nivel de presión sonora equivalente pronosticado

$L_{\text {eq exp }}$ : nivel de presión sonora equivalente experimental

RL: regresión linea

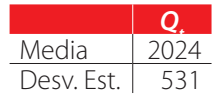

Q: flujo vehicular total

$$
\text { FIGURA 4. Comparación } L_{\text {eqexp }} \text { vs. } L_{\text {eq }} \text { RL para la Zona Centro }
$$

Efectuada la misma graficación con la Ecuación 9, se puede determinar que los datos calculados con dicha ecuación se ajustan de forma global a las condiciones de la Zona Centro, en tanto que el error oscila entre 0,1 y 0.

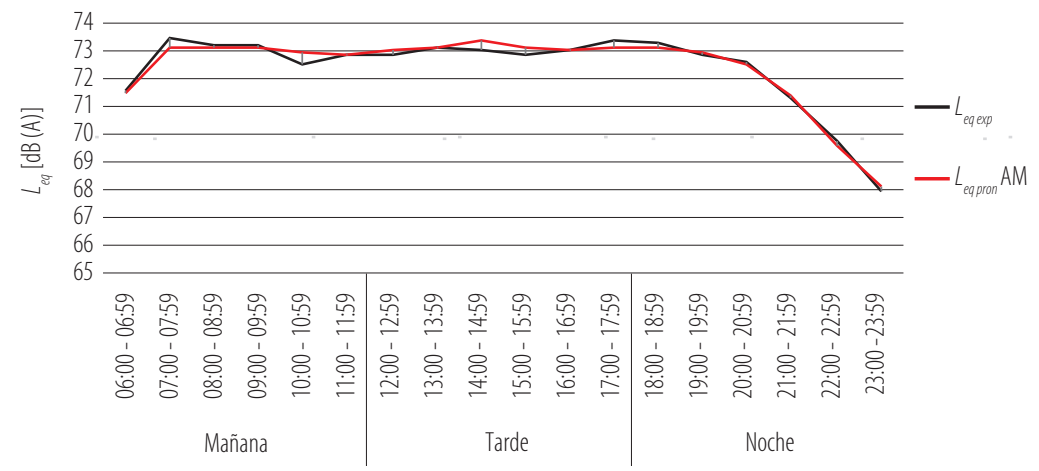

$L_{\text {eq pron }}:$ nivel de presión sonora equivalente pronosticado

$L_{\text {eq exp }}$ : nivel de presión sonora equivalente experimental

AML: análisis multivariante

\begin{tabular}{l|r|r|r} 
& $\boldsymbol{V}_{\text {prom }}$ & \multicolumn{1}{c|}{$\mathbf{Q}_{\boldsymbol{1}}$} & $\mathbf{Q}_{\boldsymbol{p}}$ \\
\hline Media & 35 & 1914 & 142 \\
\hline Desv. Est. & 3,58 & 420 & 38
\end{tabular}

Q: Flujo vehicular liviano por hora

$Q_{p}$ : Flujo vehicular pesado por hora

$V_{\text {prom }}^{p}$ :Velocidad promedio $(\mathrm{km} / \mathrm{h})$

FIGURA 5. Comparación $L_{\text {eqexp }}$ vs. $L_{\text {eq }} A M$ para la Zona Centro 


\section{Modelos predictivos de ruido para la Zona Norte}

Los resultados de la Zona Norte del DMQ, se presentan en la Ecuación 11, para la linealización y la Ecuación 11 para el análisis multivariante:

$$
\begin{gathered}
L_{\text {eqhora }}=2,63+20,12 \log Q_{t} \\
L_{\text {eqhora }}=24,12+7,13 \log Q_{1}+6,77 \log Q_{p}+0,18 V_{\text {prom }}
\end{gathered}
$$

El coeficiente de correlación de la Ecuación 6 es de 0,99 al igual que para la Ecuación 12.

A través del análisis gráfico, se nota un mayor ajuste de los datos experimentales y calculados a través de las ecuaciones 11 y 12 , esto se debe a que se cuenta con un mayor número de datos experimentales de la Zona Norte del DMQ.

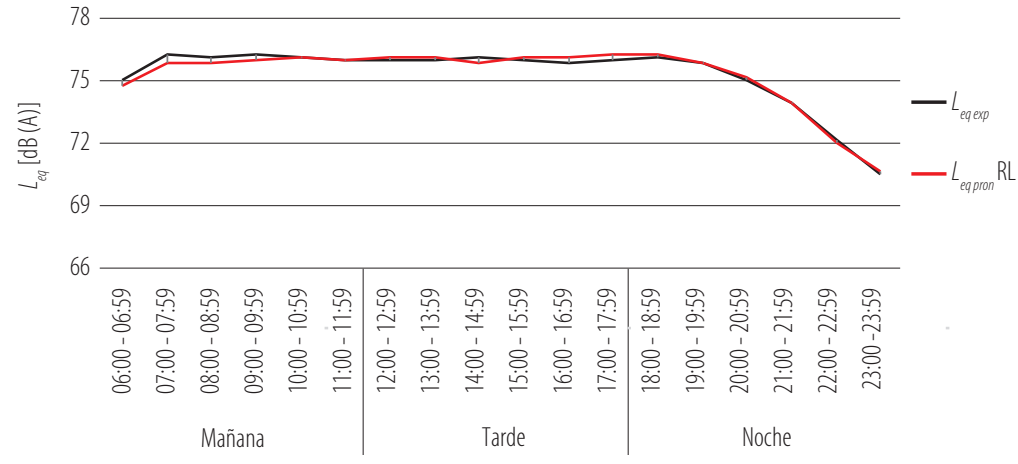

\footnotetext{
$L_{\text {eq pron }}$ : nivel de presión sonora equivalente pronosticado

$L_{\text {eq exp }}$ : nivel de presión sonora equivalente experimental

RL: regresión linea

Q: Flujo vehicular liviano por hora

Q: Flujo vehicular pesado por hora

$V_{\text {prom }}:$ Velocidad promedio $(\mathrm{km} / \mathrm{h})$
}

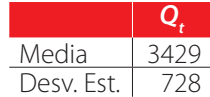

FIGURA 6. Comparación $L_{\text {eqexp }}$ vS. $L_{\text {eq }}$ RL para la Zona Norte

En el caso de la Zona Norte el número de vehículos pesados por hora $\left(Q_{p}\right)$ tiene un coeficiente de correlación de 0,96, seguido por el 0,99 del flujo vehicular liviano (Q), algo evidente sucede con la velocidad promedio $\left(V_{\text {prom }}\right)$, se trata de un valor negativo de $-0,81$, significando que la relación es inversamente proporcional. 

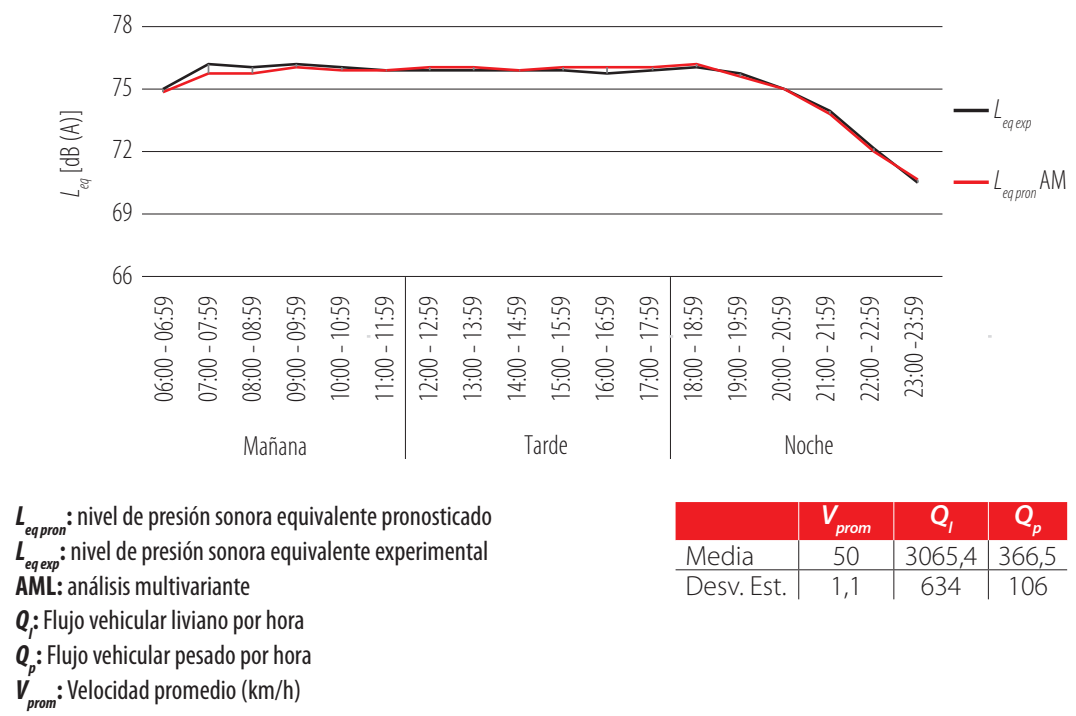

\begin{tabular}{l|c|c|c} 
& $\boldsymbol{V}_{\text {prom }}$ & $\mathbf{Q}_{\boldsymbol{I}}$ & $\mathbf{Q}_{\boldsymbol{p}}$ \\
\hline Media & 50 & 3065,4 & 366,5 \\
\hline Desv. Est. & 1,1 & 634 & 106
\end{tabular}

FIGURA 7. Comparación Leq exp Vs Leq AM para la Zona Norte

En la Figura 7 se observa que los datos calculados con la Ecuación 7 presentan valores de predicción muy cercanos a los experimentales, por lo tanto la ecuación tiene un grado muy alto de equivalencia.

\section{Modelos predictivos de ruido para el Distrito Metropolitano de Quito (DMQ)}

Los datos ingresados para el diseño del modelo estadístico integral del DMQ, fueron los promedios aritméticos de las Zonas Centro, Norte y Sur integrados en 18 valores correspondientes a cada hora a lo largo del día (06H00 a 00H00).

Para el diseño del modelo estadístico por regresión lineal simple, se usaron los datos de nivel de ruido equivalente $\left(L_{e q} d B(A)\right)$ y el logaritmo base 10 del flujo vehicular total. El coeficiente de correlación del nivel de ruido equivalente con respecto al flujo vehicular total fue 0,99 , lo que significa que existe alta correlación entre las variables. El coeficiente de determinación a diferencia del coeficiente de correlación define el grado de ajuste del modelo y este es del 0,98 para la ecuación lineal del DMQ. La ecuación obtenida por regresión lineal simple es la número (8).

$$
L_{\text {eqhora }}=23,92+14,33 \log Q_{t}
$$

El análisis multivariante se realizó con los datos promediados de las zonas sur, centro y norte de cada variable: flujo vehicular liviano y pesado, expresados en logaritmo base 10 y la velocidad promedio en $\mathrm{kmh}^{-1}$. Para diseñar la ecuación multivariante se ingresaron los mismos datos del nivel de presión sonora y los siguientes datos adicionales:

- $\quad$ Velocidad: velocidad promedio $\left(V_{\text {prom }}\right)$ 
- $\quad$ Log Q: el logaritmo base 10 del flujo vehicular liviano y pesado

- $\quad L_{e a}$ : nivel de presión sonora equivalente.

La Ecuación 14 fue obtenida y validada para el modelo de análisis multivariante para el DMQ:

$$
L_{\text {eqhora }}=9,99+13,08 \log Q_{1}+3,47 \log Q_{p}+0,26 V_{\text {prom }}
$$

El coeficiente de correlación múltiple del análisis multivariante es de 0,99, esto se explica por la cantidad de variables que ingresan en el modelo lo cual permite su mejor caracterización.

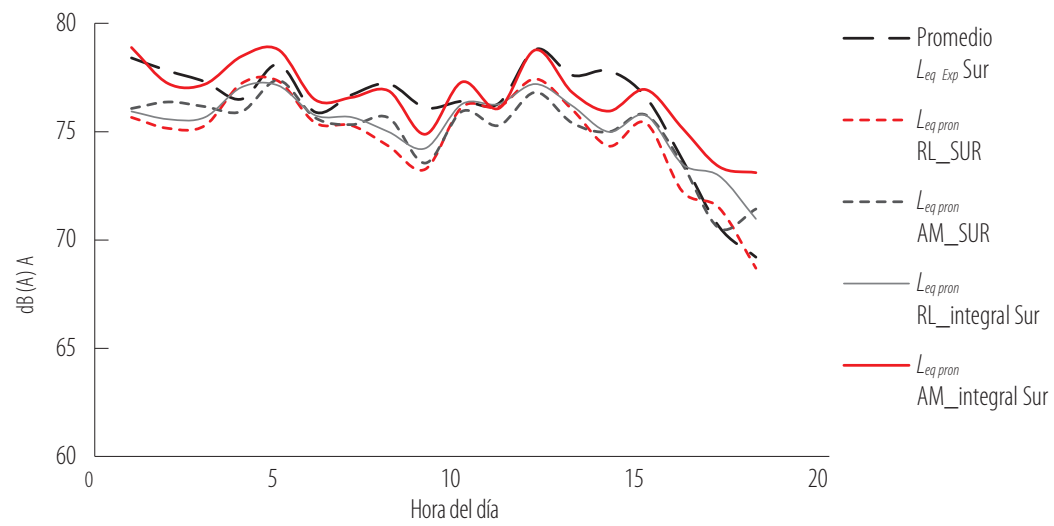

FIGURA 8. Correlación de los modelos Leq para la Zona Sur

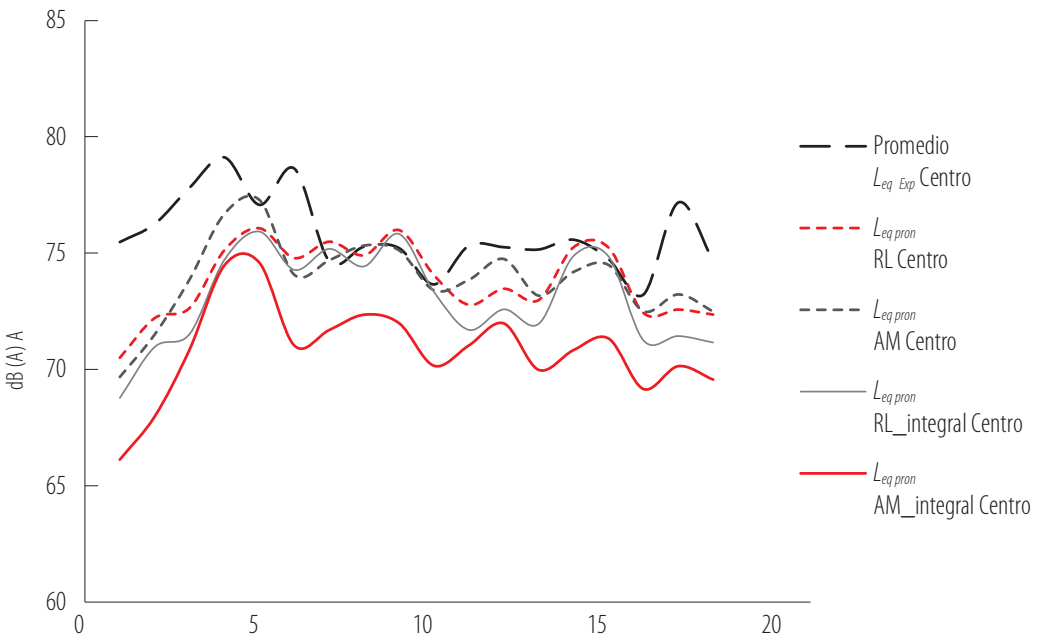




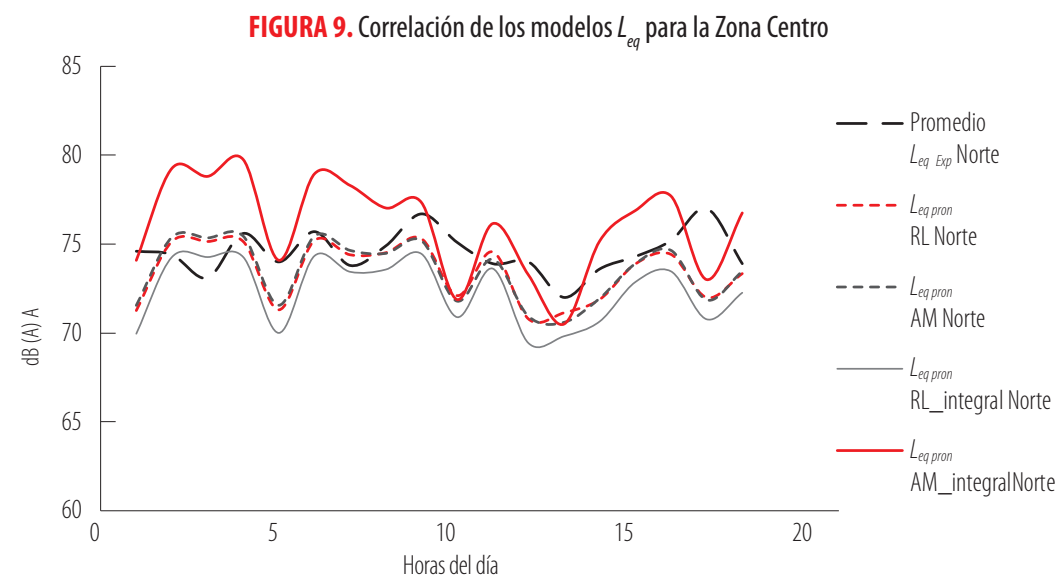

FIGURA 10. Correlación de los modelos Leq para la Zona Norte

\section{Validación por intervalos de confianza IC}

El primer proceso de validación de los modelos zonales se realizó utilizando los intervalos de confianza IC, para ello se utilizó la distribución T de Student, debido a que ésta sirve principalmente cuando se tienen muestras menores a 30 observaciones, en la presente investigación se trabajaba con 18 datos correspondientes a los promedios de monitoreos diarios de $06 \mathrm{H0O}$ a $00 \mathrm{H00}$. De esta manera se obtuvieron los valores máximo y mínimo de cada intervalo. Si la media muestral experimental se encontraba dentro de dicho intervalo de confianza, se daba por validado el modelo en el sector según corresponda. Los IC se obtuvieron a partir de la Ecuación 5 [30].

Los datos obtenidos se representan en la Tabla 1. [30-32]

TABLA 1. Validación a través de los IC

\begin{tabular}{|c|c|c|c|c|}
\hline \multicolumn{5}{|c|}{ Validación por intervalos de confianza } \\
\hline \multirow{2}{*}{ Zona } & \multicolumn{2}{|c|}{ IC simple } & \multirow{2}{*}{$\begin{array}{c}\text { Media muestreal } \\
\text { experimental }\end{array}$} & \multirow{2}{*}{ Validación } \\
\hline & Limite inferior & Limite superior & & \\
\hline Sur & 72,6 & 74,0 & 73,3 & Validado \\
\hline Centro & 72,9 & 76,3 & 74,6 & Validado \\
\hline Norte & 75,2 & 76,0 & 75,6 & Validado \\
\hline \multicolumn{5}{|c|}{ Validación por intervalos de confianza } \\
\hline \multirow{2}{*}{ Zona } & \multicolumn{2}{|c|}{ ICmultivariante } & \multirow{2}{*}{$\begin{array}{l}\text { Media muestreal } \\
\text { experimental }\end{array}$} & \multirow{2}{*}{ Validación } \\
\hline & Límiteinferior & Límite superior & & \\
\hline Sur & 72,6 & 74,0 & 73,3 & Validado \\
\hline Centro & 72,8 & 76,2 & 74,6 & Validado \\
\hline Norte & 75,2 & 76,1 & 75,6 & Validado \\
\hline
\end{tabular}




\section{Análisis ANOVA para la validación de los modelos estadísticos de ruido ambiental}

Para confirmar la validación de los modelos estadísticos se realizó el Análisis ANOVA, estableciéndose un nivel de significancia (probabilidad de cometer error tipo 1) del 5\%, a partir de aquí se calculó el estadístico crítico F, para compararlo con el estadístico F de la muestra para rechazar o no rechazar la hipótesis nula. Valores. Si F es mayor que F crítico, la hipótesis nula no se rechaza.

\begin{tabular}{|c|c|c|c|}
\hline \multicolumn{1}{|c|}{ TABLA 2. Análisis ANOVA } \\
\hline & F & Valor crítico de F & Estado \\
\hline Zona Sur & 2,54 & 2,48 & VALIDADA \\
\hline Zona Centro & 17,41 & 2,48 & VALIDADA \\
\hline Zona Norte & 9,68 & 2,48 & VALIDADA \\
\hline
\end{tabular}

La Tabla 2, determina que la combinación de las cuatro ecuaciones RL zonal, MV zonal, RL integral, MV integral; para las tres zonas del DMQ, arrojan valores de F superiores al valor crítico F establecido, validando los valores obtenidos a través de las ecuaciones estadísticas para la predicción de ruido ambiental en la ciudad.

\section{Comprobación 2019}

A partir de los datos proporcionados por la Secretaría de Ambiente del DMQ, se obtuvieron los valores promedios correspondientes a los intervalos horarios y días de muestreo realizado por la UISEK.

TABLA 3. Promedios de la Decretaría de Ambiente del DMQ año 2019

\begin{tabular}{|c|c|c|c|c|c|c|c|c|}
\hline CAMAL & Semana & $\mathbf{d} \mathbf{B} \mathbf{A}$ & CARAPUNGo & Semana & $\mathbf{d B} \mathbf{A}$ & CENTRO & Semana & $\mathbf{d B A}$ \\
\hline Enero & $14-20$ & 56,4 & Enero & $14-20$ & 58,9 & Enero & $14-20$ & 51,5 \\
\hline Febrero & $11-17$ & 54,7 & Febrero & $11-17$ & 60,7 & Febrero & $11-17$ & 52,9 \\
\hline Marzo & $18-24$ & 55,2 & Marzo & $11-17$ & 59,9 & Marzo & $11-17$ & 53,0 \\
\hline Abril & $15-21$ & 57,5 & Abril & $15-21$ & 59,2 & Abril & $15-21$ & 57,0 \\
\hline Mayo & $13-19$ & 56,0 & Mayo & $13-19$ & 59,0 & Mayo & $13-19$ & 52,4 \\
\hline Junio & $17-23$ & 55,2 & Junio & $17-23$ & 59,8 & Junio & $17-23$ & 58,2 \\
\hline Julio & $8-14$ & 55,2 & Julio & $8-14$ & 59,3 & Julio & $8-14$ & 53,1 \\
\hline Agosto & $12-18$ & 57,3 & Agosto & $12-18$ & 59,7 & Agosto & $12-18$ & 55,5 \\
\hline Septiembre & $9-15$ & 55,2 & Septiembre & $9-15$ & 59,6 & Septiembre & $9-15$ & 60,8 \\
\hline Octubre & $14-20$ & 57,3 & Octubre & $14-20$ & 59,1 & Octubre & $14-20$ & 54,5 \\
\hline Noviembre & $11-17$ & 56,6 & Noviembre & $11-17$ & 59,5 & Noviembre & $11-17$ & 57,2 \\
\hline Diciembre & $9-15$ & 57,0 & Diciembre & $9-15$ & 59,4 & Diciembre & $9-15$ & 58,1 \\
\hline
\end{tabular}

Luego se procedió a realizar la comparación con los datos obtenidos en el muestreo, del año 2019 estableciendo errores entre el valor experimental de la Secretaría y el valor de Ruido calculado a través de las ecuaciones formuladas en este trabajo. 
TABLA 4. Errores promedio de los valores de Ruido Experimental y Calculados para la zona Sur

\begin{tabular}{|c|c|c|c|c|c|c|c|c|c|c|c|}
\hline \multicolumn{12}{|c|}{ SUR • EL CAMAL } \\
\hline & $\begin{array}{c}\text { Promedio } \\
\text { Leq Exp }\end{array}$ & $\begin{array}{l}\text { Leq pron } \\
\text { RL_SUR }\end{array}$ & $\begin{array}{l}\text { Leq pron } \\
\text { AM_SUR }\end{array}$ & \begin{tabular}{|c|} 
Flujo \\
Vehicular \\
total \\
\end{tabular} & $\begin{array}{c}\text { Residuos } \\
\text { RL }\end{array}$ & $\begin{array}{c}\text { Residuos } \\
\text { AM }\end{array}$ & $\begin{array}{c}\% E \\
\text { RL-SUR }\end{array}$ & $\begin{array}{c}\% \text { EAM- } \\
\text { SUR }\end{array}$ & $\begin{array}{l}\text { Promedio } \\
\text { Velocidad }\end{array}$ & $\begin{array}{c}\text { Promedio } \\
\text { Flujo Vehicular } \\
\text { Liviano }\end{array}$ & $\begin{array}{c}\text { Promedio } \\
\text { Flujo Vehicular } \\
\text { Pesado }\end{array}$ \\
\hline & $d B(A)$ & $d B(A)$ & $d B(A)$ & Qt & $\begin{array}{l}\text { Leq exp - } \\
\text { Leqpron }\end{array}$ & $\%$ & $\%$ & $\mathrm{~km} / \mathrm{h}$ & Q & $Q p$ & \\
\hline Enero & 56,4 & 57,4 & 60,3 & 530 & $-1,0$ & $-3,9$ & 1,8 & 6,9 & 25,0 & 454 & 76 \\
\hline Febrero & 54,7 & 58,2 & 59,3 & 580 & $-3,5$ & $-4,6$ & 6,5 & 8,4 & 21,0 & 520 & 60 \\
\hline Marzo & 55,2 & 57,9 & 59,9 & 558 & $-2,7$ & $-4,7$ & 4,9 & 8,4 & 21,0 & 479 & 79 \\
\hline Abril & 57,5 & 56,5 & 58,9 & 478 & 1,0 & $-1,4$ & 1,7 & 2,4 & 21,0 & 412 & 66 \\
\hline Mayo & 56,0 & 56,9 & 58,7 & 498 & $-0,9$ & $-2,7$ & 1,6 & 4,8 & 23,0 & 448 & 50 \\
\hline Junio & 55,2 & 57,1 & 58,9 & 512 & $-1,9$ & $-3,7$ & 3,5 & 6,7 & 22,0 & 455 & 57 \\
\hline Julio & 55,2 & 58,6 & 59,4 & 604 & $-3,4$ & $-4,2$ & 6,1 & 7,6 & 21,0 & 545 & 59 \\
\hline Agosto & 57,3 & 58,5 & 60,7 & 598 & $-1,2$ & $-3,4$ & 2,1 & 5,9 & 25,0 & 523 & 75 \\
\hline Septiembre & 55,2 & 58,1 & 59,7 & 574 & $-2,9$ & $-4,5$ & 5,3 & 8,2 & 23,0 & 512 & 62 \\
\hline Octubre & 57,3 & 58,4 & 60,0 & 589 & $-1,1$ & $-2,7$ & 1,9 & 4,6 & 23,0 & 523 & 66 \\
\hline Noviembre & 56,6 & 58,9 & 58,2 & 623 & $-2,3$ & $-1,6$ & 4,0 & 2,8 & 22,0 & 589 & 34 \\
\hline Diciembre & 57,0 & 58,7 & 58,7 & 612 & $-1,7$ & $-1,7$ & 3,0 & 3,0 & 20,0 & 55 & 557 \\
\hline & & & & & $\begin{array}{l}\% \text { ERROR } \\
\text { PROMEDIO }\end{array}$ & 3,5 & 5,8 & & & & \\
\hline
\end{tabular}

TABLA 5. Errores promedio de los valores de Ruido Experimental y Calculados para la zona Centro

\begin{tabular}{|c|c|c|c|c|c|c|c|c|c|c|c|}
\hline \multicolumn{12}{|c|}{ CENTRO•GARCIA MORENOY SUCRE } \\
\hline & $\begin{array}{l}\text { Promedio } \\
\text { Leq Exp }\end{array}$ & $\begin{array}{l}\text { Leqpron } \\
\text { RL_SUR }\end{array}$ & $\begin{array}{l}\text { Leqpron } \\
\text { AM_SUR }\end{array}$ & \begin{tabular}{|c|} 
Flujo \\
Vehicular \\
total \\
\end{tabular} & $\begin{array}{l}\text { Residuos } \\
\text { RL }\end{array}$ & $\begin{array}{c}\text { Residuos } \\
\text { AM }\end{array}$ & $\begin{array}{c}\% \text { ERL- } \\
\text { SUR }\end{array}$ & $\begin{array}{l}\% E \\
\text { AM- } \\
\text { SUR }\end{array}$ & $\begin{array}{l}\text { Promedio } \\
\text { Velocidad }\end{array}$ & $\begin{array}{c}\text { Promedio } \\
\text { Flujo Vehicular } \\
\text { Liviano }\end{array}$ & $\begin{array}{c}\text { Promedio } \\
\text { Flujo Vehicular } \\
\text { Pesado }\end{array}$ \\
\hline & $d B(A)$ & $d B(A)$ & $d B(A)$ & Qt & $\begin{array}{c}\text { Leq exp - Leq } \\
\text { pron }\end{array}$ & $\%$ & $\%$ & $\mathrm{~km} / \mathrm{h}$ & Ql & Qp & \\
\hline Enero & 51,5 & 55,8 & 49,3 & 65 & $-4,3$ & 2,2 & 8,4 & 4,3 & 25,0 & 45 & 20 \\
\hline Febrero & 52,9 & 56,3 & 49,5 & 72 & $-3,4$ & 3,4 & 6,4 & 6,4 & 22,0 & 59 & 13 \\
\hline Marzo & 53,0 & 57,0 & 50,9 & 84 & $-4,0$ & 2,1 & 7,6 & 3,9 & 25,0 & 72 & 12 \\
\hline Abril & 57,0 & 57,3 & 51,9 & 89 & $-0,3$ & 5,1 & 0,6 & 8,9 & 28,0 & 65 & 24 \\
\hline Mayo & 52,4 & 56,9 & 51,5 & 82 & $-4,5$ & 0,9 & 8,6 & 1,8 & 28,0 & 63 & 19 \\
\hline Junio & 58,2 & 57,6 & 52,3 & 95 & 0,6 & 5,9 & 1,0 & 10,1 & 28,0 & 79 & 16 \\
\hline Julio & 53,1 & 57,1 & 51,6 & 85 & $-4,0$ & 1,5 & 7,5 & 2,9 & 29,0 & 75 & 10 \\
\hline Agosto & 55,5 & 56,7 & 51,4 & 78 & $-1,2$ & 4,1 & 2,1 & 7,3 & 30,0 & 65 & 13 \\
\hline Septiembre & 60,8 & 56,9 & 51,4 & 82 & 3,9 & 9,4 & 6,4 & 15,4 & 28,0 & 65 & 17 \\
\hline Octubre & 54,5 & 58,4 & 51,8 & 110 & $-3,9$ & 2,7 & 7,1 & 4,9 & 20,0 & 92 & 18 \\
\hline Noviembre & 57,2 & 58,0 & 51,3 & 103 & $-0,8$ & 5,9 & 1,5 & 10,4 & 20,0 & 90 & 13 \\
\hline Diciembre & 58,1 & 59,0 & 52,3 & 125 & $-0,9$ & 5,8 & 1,5 & 10,0 & 18,0 & 105 & 20 \\
\hline & & & & & $\begin{array}{l}\text { \% ERROR } \\
\text { PROMEDIO }\end{array}$ & 4,9 & 7,2 & & & & \\
\hline
\end{tabular}


TABLA 6. Errores promedio de los valores de Ruido Experimental y Calculados para la zona Norte

\begin{tabular}{|c|c|c|c|c|c|c|c|c|c|c|c|}
\hline \multicolumn{12}{|c|}{ NORTE • CARAPUNGO } \\
\hline & $\begin{array}{c}\text { Promedio } \\
\text { Leq Exp }\end{array}$ & $\begin{array}{l}\text { Leq pron } \\
\text { RL_SUR }\end{array}$ & $\begin{array}{l}\text { Leqpron } \\
\text { AM_SUR }\end{array}$ & $\begin{array}{c}\text { Flujo } \\
\text { Vehicular } \\
\text { total }\end{array}$ & $\begin{array}{c}\text { Residuos } \\
\text { RL }\end{array}$ & $\begin{array}{c}\text { Residuos } \\
\text { AM }\end{array}$ & $\begin{array}{c}\% E \\
\text { RL-SUR }\end{array}$ & $\begin{array}{c}\text { \%EAM- } \\
\text { SUR }\end{array}$ & $\begin{array}{l}\text { Promedio } \\
\text { Velocidad }\end{array}$ & $\begin{array}{c}\text { Promedio } \\
\text { Flujo Vehicular } \\
\text { Liviano }\end{array}$ & $\begin{array}{c}\text { Promedio } \\
\text { Flujo Vehicular } \\
\text { Pesado }\end{array}$ \\
\hline & $d B(A)$ & $d B(A)$ & $d B(A)$ & Qt & $\begin{array}{c}\text { Leq exp - Leq } \\
\text { pron }\end{array}$ & $\%$ & $\%$ & $\mathrm{~km} / \mathrm{h}$ & Ql & $Q p$ & \\
\hline Enero & 58,9 & 60,8 & 60,3 & 250 & $-1,9$ & $-1,4$ & 3,1 & 2,4 & 35,0 & 185 & 65 \\
\hline Febrero & 60,7 & 59,4 & 58,9 & 195 & 1,3 & 1,8 & 2,2 & 2,9 & 38,0 & 175 & 20 \\
\hline Marzo & 59,9 & 60,8 & 60,1 & 252 & $-0,9$ & $-0,2$ & 1,5 & 0,4 & 33,0 & 169 & 83 \\
\hline Abril & 59,2 & 60,3 & 59,6 & 232 & $-1,1$ & $-0,4$ & 1,9 & 0,6 & 35,0 & 145 & 87 \\
\hline Mayo & 59,0 & 60,9 & 60,2 & 256 & $-1,9$ & $-1,2$ & 3,2 & 2,0 & 30,0 & 178 & 78 \\
\hline Junio & 59,8 & 59,8 & 59,5 & 212 & 0,0 & 0,3 & 0,0 & 0,6 & 35,0 & 160 & 52 \\
\hline Julio & 59,3 & 60,3 & 59,9 & 229 & $-1,0$ & $-0,6$ & 1,6 & 1,0 & 35,0 & 182 & 47 \\
\hline Agosto & 59,7 & 59,2 & 59,0 & 189 & 0,5 & 0,7 & 0,9 & 1,2 & 38,0 & 154 & 35 \\
\hline Septiembre & 59,6 & 59,6 & 59,3 & 204 & 0,0 & 0,3 & 0,0 & 0,5 & 37,0 & 174 & 30 \\
\hline Octubre & 59,1 & 61,1 & 60,7 & 265 & $-2,0$ & $-1,6$ & 3,4 & 2,7 & 35,0 & 206 & 59 \\
\hline Noviembre & 59,5 & 60,4 & 60,0 & 235 & $-0,9$ & $-0,5$ & 1,5 & 0,8 & 33,0 & 185 & 50 \\
\hline Diciembre & 59,4 & 61,2 & 60,7 & 270 & $-1,8$ & $-1,3$ & 3,0 & 2,1 & 33,0 & 195 & 75 \\
\hline & & & & & $\begin{array}{l}\text { \% ERROR } \\
\text { PROMEDIO }\end{array}$ & 1,9 & 1,4 & & & & \\
\hline
\end{tabular}

Los datos de ruido medidos por la Secretaría del Ambiente, al corresponder a lugares con poca circulación, son inferiores a los obtenidos experimentalmente por la UISEK en años precedentes en las vías con alto y mediano tránsito vehicular, a pesar de ello, el modelo presenta residuales que al ser transformados en \% de error presentan valores comprendidos entre 1.4 y $7.2 \%$. Acorde con González y Domínguez, las mediciones de ruido ambiental acarrean errores de hasta un $15 \%$ dependiendo de las circunstancias de medición, por lo que los datos calculados podrían considerarse admisibles para su uso y aplicación en planificación y calidad ambiental.

\section{CONCLUSIONES}

De manera general, del análisis de los datos obtenidos, se puede establecer que el nivel de ruido ambiental en la Zona Sur es más alto que en la Centro y esta a su vez es superior a la de la Zona Norte, esto se corrobora con los datos medidos in situ a lo largo del periodo de investigación. La actualización del año 2019 en el punto El Camal, registra que la aplicación de los modelos suministra valores ligeramente superiores, con residuales para la regresión lineal de 3,5 \% y para el análisis multivariante de 5,8\%. Por lo que se aprecia la necesidad de ajustar el modelo con la intención de minimizar estos residuales para la zona Sur. 
Hay mayor correspondencia de ruido ambiental entre las Zonas Sur y Norte, en la Zona Centro es algo menor, esto puede deberse a varias razones, entre ellas la morfología de la ciudad que es más compleja en el centro de Quito, el tipo de actividades que se desarrollan, además de que las características urbanas de la zona plantean superficies resonantes de ruido, variabilidad en el uso automotor y comercio callejero permanente. La actualización del año 2019, y debido al cambio de circulación en el Centro de la Ciudad de Quito, permitió validar el hecho de que para menor número de vehículos el ruido ambiental disminuye, pero también permitió verificar que un menor número de automóviles desplazándose, generan mayores residuales en los modelos versus datos experimentales, así para el modelo de regresión lineal, se obtuvo un \%E de 4,9\% y para el modelo de análisis multivariante, el error presentado fue el más alto, 7,2 \%.

A través del análisis de intervalos de confianza se llegó a una primera validación de las ecuaciones zonales, esta validación se ratificó con el análisis ANOVA; las dos validaciones permiten inferir que la predicción de ruido ambiental por tráfico rodado, a través de las ecuaciones del presente estudio, posibilitarán predecir a bajos costos los niveles de contaminación acústica por fuentes móviles en el DMQ. Al no tomar en cuenta variables como fachadas, alturas de los edificios, pendientes de las rutas, etc., estas ecuaciones se convierten en una herramienta sencilla de aplicar, bastando realizar un conteo de automóviles livianos, pesados y totales, así como la velocidad de los mismos, lo que con el avance tecnológico actual, se lo puede realizar a través de una cámara de video sencilla. Esto permitirá a los entes encargados de la Planificación Territorial y de la calidad ambiental, predecir los impactos ambientales de la ubicación de nuevos proyectos arquitectónicos, de salud, industriales o sociales.

El análisis ANOVA, confirmó lo evidenciado gráficamente, esto es, que las ecuaciones de predicción particulares funcionan al igual que las generales para la ciudad de Quito, ya que todas ellas superaron el valor crítico $F$, indicando que se encuentran en la zona de aceptación de la predicción y por tanto pudiendo ser usadas indistintamente. En el año 2019 no se pudo validar la ecuación general para el distrito, por lo que se hace necesario una actualización de datos de ruido ambiental a lo largo y ancho de la ciudad de Quito. Los valiosos datos de la Secretaria de Ambiente del DMQ, podrían ser tratados con la metodología aquí descrita para obtener correlaciones sencillas, que a futuro les permitan mejorar la calidad ambiental acústica del Distrito.

Las dos ecuaciones zonales RL y MV se ajustan a los valores experimentales obtenidos, como se puede apreciar en las Figuras 2, 3, 4, 5, 6, 7. El comportamiento del ruido a lo largo del día en los tres sectores es uniforme en horas de la mañana y de la tarde, presentando promedios de $72,0 \mathrm{~dB}(\mathrm{~A})$ y $75,9 \mathrm{~dB}(\mathrm{~A})$, mientras que en la noche disminuye la intensidad notoriamente, hasta tener niveles de ruido de $68,0 \mathrm{~dB}(\mathrm{~A})$, este comportamiento permite ajustar de mejor manera el modelo integral de regresión lineal simple.

Las variables que se han venido tomando entre flujo vehicular, nivel de ruido y velocidad, son las que mayor grado de correlación manifiestan, esto significa, que con estas tres variables, los modelos predictivos de ruido para el Distrito Metropolitano de Quito son confiables para representar la realidad acústica del lugar, lo que permitirá su aplicación en la planificación y ordenamiento territorial de la ciudad. Las variables utilizadas en el diseño de los modelos estadísticos son económicas de cuantificarlas, son propias de 
la ciudad de Quito y no modelos adaptados que presentan una mayor incertidumbre. Al ser resultados de datos tomados en diferentes años, lugares, días y horas, dentro de la ciudad, son modelos únicos y propios para las condiciones de Quito, pero además son simples, de aplicación sencilla y barata, pudiendo convertirse en una herramienta esencial para Estudios de Evaluación de Impacto Ambiental ex ante, en el Distrito.

El análisis de las variables tanto dependientes $\left(\mathrm{L}_{\text {eq }}\right)$ como independientes $\left(\mathrm{V}_{\text {prom }}, \mathrm{Q}_{\mathrm{t}^{\prime}} \mathrm{Q}_{\mathrm{I}}\right.$ y $Q_{p}$ ) permitió identificar la relación existente entre el nivel de ruido equivalente y el flujo vehicular. El coeficiente de correlación de las ecuaciones estadísticas, en promedio para cada zona, es de 0,99, significando que la predicción que se realice a través de las mismas se ajustará al ruido ambiental de las diferentes zonas del Distrito. Ajuste que con la verificación realizada en el 2019, permite su utilización tres años después de generada. Se recomienda anualmente validar las ecuaciones comparando los datos de ruido medidos por la Secretaría de Ambiente con datos resultado de las ecuaciones con las variables medidas, ampliando esta vez la validación a la estación Jipijapa.

El comportamiento del ruido a lo largo del día en los tres sectores, es uniforme en horas de la mañana y de la tarde, presentando valores promedios de $72,0 \mathrm{~dB}(\mathrm{~A})$ y $75,9 \mathrm{~dB}(\mathrm{~A})$, mientras que en la noche disminuye la intensidad notoriamente hasta tener niveles de ruido de $68,0 \mathrm{~dB}(\mathrm{~A})$, los modelos que mejor se ajustan a cada Zona son los siguientes:

- Zona Sur: Modelo zonal Multivariante; Modelo Integral Simple.

- Zona Centro: Modelo zonal Simple, Modelo Zonal Multivariante, Modelo Integral Simple.

- Zona Norte: Modelo zonal Multivariante, Modelo Integral Simple.

- $\quad$ DMQ: Modelo Integral Simple, Modelo Integral Multivariante.

En términos generales, el mejor ajuste es el Lineal Simple para el modelo estadístico integral de la ciudad, en tanto que el modelo Multivariante se ajusta mejor a los valores zonales, tal como se aprecia en las Figuras 8, 9 y 10. La corroboración del año 2019, ratifica lo indicado, ya que los residuales para Norte, Centro, Sur para el ajuste lineal son menores que para el análisis multivariante. Tablas 3,4,5.

En el entendido de que todas las ciudades deben disponer de un documento legal que permita mejorar la gestión de la contaminación acústica por tráfico rodado, enfocado a un desarrollo sustentable óptimo, relacionando la calidad de vida con el uso de los recursos de movilización, los ocho modelos de predicción de ruido servirán indistintamente de soporte a la normativa que establezca a este respecto la autoridad de la ciudad y su respectivo control. Al comprobarse su validez al año 2019, su uso permitirá una mayor facilidad para predecir valores futuros con ajustes del $5 \%$ trianual, al actual crecimiento vehicular del DMQ.

Los modelos tienen la ventaja de que utilizan variables y obtienen resultados numéricos de fácil compresión y por ende difusión y aplicación, pudiendo ser una herramienta de concienciación de la población capitalina, de aplicación en EIAs ex ante y Expost, y principalmente como instrumento de regulación vehicular con fines de encontrar un mayor confort acústico para los habitantes del Distrito. 


\section{AGRADECIMIENTOS}

Un agradecimiento muy especial a la Ing. Valeria Díaz MSc, de la Secretaría de Ambiente del Distrito Metropolitano de Quito por su apoyo incondicional y la entrega de los datos horarios de Ruido Ambiental para la realización de la comparación con el año 2019.A los estudiantes Daniela López y Andrés Coral de la Carrera de Ingeniería Ambiental de la UISEK por su ayuda en el monitoreo de variables realizado a lo largo del 2019. Por último, queremos dejar constancia de nuestro agradecimiento a todos y cada uno de los Ingenieros Ambientales de la UISEK que desde el 2009 trabajaron en la recolección, mapeo, y procesamiento de datos y variables para la determinación del ruido ambiental del DMQ.

\section{CONTRIBUCIONES DE LOS AUTORES}

Tania Moromenacho: curación de datos para la investigación, incluyendo las labores de campo, experimentales y estadísticas.

Alonso Moreta: análisis estadístico, desarrollo y validación de las ecuaciones de predicción de ruido.

Fabio Villalba: concepción de la investigación, análisis e interpretación de los datos, revisión crítica del contenido intelectual del manuscrito.

Jorge Esteban Oviedo: concepción de la investigación, diseño de la metodología, análisis e interpretación de los datos, validación y verificación de los resultados, supervisión del monitoreo de variables del año 2019.

Katty Coral: concepción de la investigación, diseño de la metodología; administración del proyecto, análisis e interpretación de los datos, validación y verificación de los resultados, redacción del manuscrito, comparación año 2019. 


\section{REFERENCIAS}

[1] Organización Mundial de la Salud. (2003). Guías para el Ruido Urbano: 1-20.

[2] García, B., Garrido F (2003). La contaminación acústica en nuestras ciudades. La Caixa: 169-194.

[3] Cavas L., Caballero J., MuñozV. (2006). Análisis comparativo de un modelo teórico de mediciones sonoras y el software SOUNPLAN Ver. 6,2 aplicado al tráfico vehicular. Revista de Ingeniería Universidad de los Andes. 23(1): 58-67

[4] Berglunt, B. (1999). Guía para el ruido urbano. Centro Panamericano de Ingeniería Sanitaria y Ciencias del Ambiente, OPS/CEPIS.

[5] Álvarez, J., \& Suárez, E. (2008). Estudio Comparativo de modelos de predicción de ruido de tráfico rodado utilizando mediciones en la ciudad de Osorno. Valdivia: Universidad Austral de Chile

[6] Lombeida M, (2012). Realización de un modelo matemático predictivo de ruido urbano, para la ciudad de Quito, basado en el modelo de predicción de Sánchez. Universidad Internacional SEK, Quito.

[7] Moreno G, (2012). Realización de un modelo matemático predictivo para ruido urbano de la ciudad de Quito y comparación con el modelo CoRTN. Universidad Internacional SEK, Quito.

[8] Agencia Metropolitana de tránsito AMT. (20 de enero de 2015). Obtenido de http://www.amt.gob.ec/index.php/ pico-placa-homepage.html

[9] González Alice. (2005). Evaluación de impacto acústico:Modelos predictivos sencillos que podrían dar complicaciones. UDELAR: 1-12

[10] Municipio del Distrito Metropolitano de Quito (2000). Plan General de Desarrollo Territorial del DMQ: 7-15.

[11] Genaro, N. (2014). Sistema de predicción de ruido urbano mediante redes neuronales. Universidad de Granada: 21-29.

[12] Rojo, M. (2007). Regresión Lineal Múltiple. Instituto de Economía y Geografía: 2-25.

[13] Recio, A., Carmona, R., Linares, C., Ortiz, C., Banegas, J.R., Díaz, J. (2016) Efectos del ruido urbano sobre la salud: estudios de análisis de series temporales realizados en Madrid. Instituto de Salud Carlos III, Escuela Nacional de Sanidad: 5-25.

[14] Andrade C, (2011), Elaboración de mapa de ruido de la red vial del Distrito Metropolitano de Quito: zona norte 1.(Tesis de pregrado en Ingeniería Ambiental) Universidad Internacional SEK. Quito.

[15] Izurieta A, (2009) Elaboración de un mapa de ruido ambiental y estudio de factibilidad de la ubicación de los puntos de monitoreo para la red de monitoreo de ruido ambiental en el Distrito Metropolitano de Quito, zona 4. (Norte de Quito). (Tesis de pregrado en Ingeniería Ambiental) Universidad Internacional SEK. Quito.

[16] Rojas C, (2010), Diseño del mapa de ruido ambiental de los sectores: Cofavi, Solca, Jipijapa, Estación Norte y Belisario en el Distrito Metropolitano de Quito, Provincia de Pichincha - Ecuador. (Tesis de pregrado en Ingeniería Ambiental) Universidad Internacional SEK. Quito.

[17] Díaz F, (2009), Elaboración de un mapa de Contaminación acústica del Distrito Metropolitano de Quito - Sur, Utilizando Sistemas de Información Geográfica. (Tesis de pregrado en Ingeniería Ambiental) Universidad Internacional SEK. Quito.

[18] Rubianes F, (2009), Elaboración de un mapa de ruido ambiental para determinar la ubicación más apropiada de los puntos de monitoreo para la Red Mínima de Monitoreo del Ruido Ambiental en el Distrito Metropolitano de Quito, Zonas 2: Calderón, Carapungo, Centro, Los Chillos y Tumbaco. (Tesis de pregrado en Ingeniería Ambiental) Universidad Internacional SEK. Quito.

[19] Amores, J, (2010), Elaboración de un mapa de ruido del Distrito Metropolitano de Quito - Zona Sur. (Tesis de pregrado en Ingeniería Ambiental) Universidad Internacional SEK. Quito

[20] Vazquez, N, (2009), Elaboración de un mapa de ruido ambiental y estudio de factibilidad para la ubicación de los puntos de monitoreo de la red de monitoreo ambiental en el Distrito Metropolitano de Quito, zona norte. (Tesis de pregrado en Ingeniería Ambiental) Universidad Internacional SEK. Quito.

[21] Ochoa, W, (2011) Generación del mapa de ruido de la red vial del anillo urbano del Distrito Metropolitano de Quito, zona centro. (Tesis de pregrado en Ingeniería Ambiental) Universidad Internacional SEK. Quito. 
[22] Mora, P, (2010) Diseño de un mapa de ruido de la contaminación acústica de la zona urbana norte (Carapungo, Calderón, Cotocollao, La Delicia, Pablo Arturo Suárez) de la Ciudad de Quito. (Tesis de pregrado en Ingeniería Ambiental) Universidad Internacional SEK. Quito.

[23] Peña, E, (2011) Elaboración de un mapa de ruido de la red vial del Distrito Metropolitano de Quito, zona Centro Norte. 2010 - 2011. (Tesis de pregrado en Ingeniería Ambiental) Universidad Internacional SEK. Quito.

[24] Salazar, D, (2011) Elaboración de un mapa de ruido del Distrito Metropolitano de Quito- zona norte 2. (Tesis de pregrado en Ingeniería Ambiental) Universidad Internacional SEK. Quito.

[25] Villafuerte, D, (2011) Elaboración de un mapa de ruido del Distrito Metropolitano de Quito, zona sur, Quito. (Tesis de pregrado en Ingeniería Ambiental) Universidad Internacional SEK. Quito.

[26] Velez, M (2013) Validación de los modelos matemáticos de ruido urbano UISEK de linealización de una función no lineal y análisis multivariante en el sector centro occidente de la ciudad de Quito. (Tesis de pregrado en Ingeniería Ambiental) Universidad Internacional SEK. Quito.

[27] Palacios W (2013). Validación de los modelos matemáticos predictivos de ruido urbano UISEK por análisis multivariante y linealización de una funcion no lineal en la zona noroccidental de Quito. (Tesis de pregrado en Ingeniería Ambiental) Universidad Internacional SEK. Quito.

[28] Kattan F (2013), Validación de los modelos matemáticos de ruido urbano UISEK de linealización de una función no lineal y análisis multivariante en el sector suroriental de la ciudad de Quito. (Tesis de pregrado en Ingeniería Ambiental) Universidad Internacional SEK. Quito.

[29] Padron R (2014), Validación de modelos matemáticos de linealización de una función no lineal y análisis multivariante para ruido ambiental urbano en la zona Nor-Occidente de Quito. (Tesis de pregrado en Ingeniería Ambiental) Universidad Internacional SEK. Quito.

[30] Ballesteros C (2014), Validación de los modelos matemáticos predictivos de ruido urbano por linealización de una función no lineal y análisis multivariante en la zona centro occidente de quito para el año 2014. (Tesis de pregrado en Ingeniería Ambiental) Universidad Internacional SEK. Quito.

[31] Escobar, A (2015), Validación de los modelos matemáticos de ruido urbano UISEK de linealización de una función no lineal y análisis multivariante en el sector sur oriental de la ciudad de Quito. (Tesis de pregrado en Ingeniería Ambiental) Universidad Internacional SEK. Quito.

[32] Tapia F, (2015). Validación de modelos matemáticos predictivos de ruido urbano UISEK a través de la linealización de una función no lineal y análisis multivariante en el sector noroccidente de Quito. (Tesis de pregrado en Ingeniería Ambiental) Universidad Internacional SEK. Quito.

[33] Secretaria del Ambiente. (2015). Obtenido de http://www.quitoambiente.gob.ec/index.php?option=com_ k2\&view=item\&layout=item\&id=37\&ltemid=18\&lang=es, Abril 2016

[34] Instituto Ecuatoriano de Estadísticas y Censos (2019), Anuario Estadístico de transporte. Quito.

[35] Ecuador Tv, la televisión del estado (2019). Obtenido de: https://shorturl.at/ntKW6, Noviembre 2019

[36] Ramírez González A, Domínguez E, (2011). El ruido vehicular urbano: problemática agobiante de los países en vías de desarrollo. Revista de la Academia Colombiana de Ciencias Exactas, Físicas y Naturales, vol.35 no.137 Bogotá Oct./Dec. 2011

[37] Código Municipal para el Distrito Metropolitano de Quito (2019)

[38] MAE, Ministerio de Ambiente del Ecuador, (2008). Texto Unificado de Legislación Ambiental Secundaria.

[39] MAE, Ministerio de Ambiente del Ecuador, (2015). Acuerdo Ministerial 097 A. 